\title{
From an Overcrowded to a Smoothly Functioning Emergency Department within Two Months
}

Fuad Basis*

Deputy Director Medical and Research Development, Israel

*Corresponding author: Fuad Basis, Deputy Director Medical and Research Development, Israel, Tel: +972-502061402; E-mail: f_basis@rambam.health.gov.il

Received date: January 16, 2017; Accepted date: January 25, 2017; Published date: February 06, 2017

Copyright: (c) 2017 Basis F. This is an open-access article distributed under the terms of the Creative Commons Attribution License, which permits unrestricted use, distribution, and reproduction in any medium, provided the original author and source are credited.

\begin{abstract}
Background: Our Emergency Department (ED) went through two periods with different managers. Although the number of beds, visits and admissions during these two periods didn't change significantly, a change in the ED's policy, without a change in the hospital's policy, converted a very crowded ED to an uncrowded one within few weeks, and changed the pattern of work in the hospital, gradually for many years.
\end{abstract}

Objectives: To share our experience and describe how a very crowded ED was transformed into an uncrowded one within few weeks and how we maintained the change thereafter.

Methods: All data concerning the ED and other department activities were collected by using our hospital's computerized business intelligence $(\mathrm{BI})$ system.

Results: The average length of stay (LOS) of admitted patients in the ED was $5.05+0.98$ hrs during period "A" (1993-2001). It dropped to 2.45+0.21 during period "B" $(2002-2007)(P<0.001)$. The percent of patients boarding in the ED more than 10 hours dropped from $11.5+2.4 \%$ (period "A") to $2.6+0.7 \%$ (period "B") within three months. Despite a gradual increase in the number of admissions to the GM wards, there was a gradual decrease in the average LOS during period "B", compared to period "A" (6.1 vs. 5.5 days) $(P=0.001)$. The death rate among patients in the ED and in the GM wards decreased significantly during period "B" $(P<0.001)$.

Conclusions: We were able to achieve a difficult goal of eliminating overcrowding in the ED and consequently, other hospital's wards only through assertiveness, persistence, adherence to protocols and the full personal backing of the hospital's manager.

Keywords: Emergency medicine; Crowding; Length of stay; Policy; Internal medicine

\section{Introduction}

The assumption that an overcrowded ED is not an ED failure but rather a whole hospital failure with most causes beyond the control of the emergency departments, has become a consensus among many emergency physicians [1,2].

We have 4 General Medicine (GM) wards in the hospital with a total official count of 164 beds. The internal medicine departments are not differentiated on the basis of urgency or acuity. Rather at our institution the internal medicine departments to date have been maintained with a high degree of uniformity according to these categories. When the individual GM wards are full, additional 5 patients to 6 patients are admitted and temporarily placed in the ward's hallways. From 1993 till 2007 our Emergency Department (ED) passed two distinct periods: (Period "A"), from 1993 till 2001, during which the ED was very crowded, averaging $16+3$ patients boarding in the ED waiting for inpatient beds each morning. The second period (Period "B"), was from 2002 till 2007, during which the ED was smoothly functioning averaging $3+1$ patients boarding in the ED. During each period the ED was managed by a different director. In both periods "A" and "B", the hospital General Director urged the ED director to move patients from the ED within 4 hrs. After a decision for admission was made. However, the crowding situation in the ED in each period was very different.

Since all data has been computerized by the same system from 1998 till 2005, we have an opportunity to examine how the functioning of the GM wards influenced ED overcrowding and vice versa. In this study, we would like to show how a change in ED policy and function can transform a crowded ED to a smoothly running ED within a few weeks.

There are some studies which have examined the issue of ED overcrowding, and suggested many ways to solve the problem [3-5], but we did not find, so far, studies that had examined the abrupt change in the ED policy into zero tolerance of boarding patient in the ED, within such a short time. We did not find either its effect on the length of stay (LOS) in GM wards and on ED overcrowding, without changing the number of hospital beds, the staff number, or the inpatient flow to the ED.

\section{Objectives}

To describe how we transformed a very crowded ED into an uncrowded and smoothly functioning ED within a few weeks, and how we maintained the change thereafter. 
Page 2 of 6

\section{Materials and Methods}

This is a descriptive observational retrospective study. The data from 1998 till 2005 was collected using the same parameters thus allowing a comparison study. From 2005 on, all activities of the hospital's departments have been computerized using a new business intelligence (BI) system. Not all data collected before 2005 can be compared to what is available today, confining our study to four years before and four years after a policy change took place in the ED.

We used the T-test for the comparison study and for the statistical analysis and a chi square test for categorical variances. We also calculated the medians where the LOS included a high range of variables.

At the end of 2001, the manager of the ED, a specialist in internal medicine retired and was replaced by a specialist in emergency medicine (EM). His deputy was a specialist in EM and internal medicine. The new team redefined the role of the $\mathrm{ED}$, according to guidelines found in emergency medicine literature, into a place where patients are specifically evaluated for urgent conditions, using a minimum workup needed to make safe decisions for admission or discharge. It is important to clarify that the changes in policy and accompanying outcomes occurred without planned changes in ED human resources.

For that purpose, the following steps were done:

New protocols and guidelines for workups were constructed.

The protocols were tightly implemented by the ED managers during all shifts.

New protocols for blood tests, blood cultures and imaging facilities were created as well.

The senior nursing staff was instructed to maintain efficiency and to involve the managers when patients' workups were delayed.

Constant rounds were performed every two hours by the shift's chief physician, the chief nurse and the residents. During these rounds, decisions were made and patients with delayed workup were pinpointed and treated more efficiently.

Non urgent admissions from the hospital's outpatient clinics and elective admissions were no longer allowed to pass through the ED.
A computerized program, later called a "Justice Table", was designed to spread the patients evenly between the departments, taking into account the complexity of the patient's ailment.

When a certain department had few discharges compared to admissions, it was temporarily closed for admissions for two days and admitted patients were shifted to other departments. On the third day, that department admitted extra patients that it "owed" other departments. Periodically, we verified that the "Justice Table" was still balanced.

The staff was instructed to move patients to the medical wards within no more than 4 hours after a decision for admission was made.

From time to time, the number of patients boarding in the GM ward hallways reached 8 patients during weekends, instead of 5, reminding the staff to be more efficient.

During the night shifts, the hospital managers on call were instructed to adhere to the rules implemented by the hospital manager.

To facilitate the consultation process, consultants from other fields were called by the ED physicians themselves. The intervention of the chief physician or the ED manager was sought by the chief nurse in cases of significant delays in the consultation process (more than 1.5 hrs.). The hospital manager's deputies were involved urging consultants and preventing delays.

\section{Results}

During period "A" (from 1998 till 2001) the actual number of beds in the medical wards was 185 beds on average (range: 180 to 189). During period "B" (2002 till 2005) the average was 184 beds see Table 1 .

The change in the number of beds between both periods is statistically non-significant $(\mathrm{P}>0.5)$. During period " $\mathrm{A}$ " the number of admissions to the GM wards decreased gradually by $11.8 \%(18,149$ to $16,002)$ year by year, while during period "B" it increased gradually by $8.1 \%(16,778$ to 18,149$)$. However, the differences between the total number of admissions to the GM department during the two periods (A and $\mathrm{B}$ ) was statistically non-significant $(\mathrm{P}=0.24)$ (Table 1$)$.

\begin{tabular}{|c|c|c|c|c|c|c|c|c|}
\hline \multicolumn{5}{|l|}{ Period A } & \multicolumn{4}{|l|}{ Period B } \\
\hline Year & 1998 & 1999 & 2000 & 2001 & 2002 & 2003 & 2004 & 2005 \\
\hline No' visits to Medical ED & 48235 & 48978 & 48278 & 48670 & 46895 & 49366 & 48161 & 50334 \\
\hline $\begin{array}{l}\text { Admission to } \mathrm{GM} \\
\text { departments }\end{array}$ & 17392 & 16993 & 16287 & 16002 & 16778 & 17697 & 17385 & 18149 \\
\hline Actual no Beds & 184 & 184 & 184 & 184 & 180 & 187 & 189 & 184 \\
\hline $\begin{array}{l}\text { LOS in GM Division } \\
\text { (days) }\end{array}$ & 5.9 & 5.6 & 6.1 & 6.6 & 6.3 & 5.8 & 5.2 & 5.3 \\
\hline $\begin{array}{l}\text { Average LOS in ED after } \\
\text { decision for admission } \\
\text { (hours) }\end{array}$ & 5.5 & 4.3 & 5.6 & 6.7 & 2.8 & 3.1 & 3.3 & 3.2 \\
\hline $\begin{array}{l}\% \text { staying }>10 \text { hrs in ED } \\
\text { each morning }\end{array}$ & $8 \%$ & $10 \%$ & $13 \%$ & $15 \%$ & $3.20 \%$ & $2.50 \%$ & $1.80 \%$ & $2.10 \%$ \\
\hline
\end{tabular}


Citation: Basis F (2017) From an Overcrowded to a Smoothly Functioning Emergency Department within Two Months. J Gen Pract (Los Angel)

Page 3 of 6

\begin{tabular}{|c|c|c|c|c|c|c|c|c|}
\hline $\begin{array}{l}\% \text { Readmissions } 1 \text { week } \\
\text { into GM departments }\end{array}$ & $5.51 \%$ & $4.60 \%$ & $4.02 \%$ & $4.80 \%$ & $4.75 \%$ & $5.25 \%$ & $5.21 \%$ & $4.88 \%$ \\
\hline $\begin{array}{l}\text { Number Readmissions } 1 \\
\text { week into GM } \\
\text { departments }\end{array}$ & 545 & 449 & 395 & 439 & 453 & 531 & 501 & 503 \\
\hline Death rates in ED (\%) & 0.11 & 0.15 & 0.11 & 0.2 & 0.08 & 0.06 & 0.09 & 0.08 \\
\hline No' deaths in ED & 53 & 73 & 53 & 97 & 37 & 29 & 43 & 40 \\
\hline Death rate in $\mathrm{GM}$ division & $8.82 \%$ & $8.36 \%$ & $9.24 \%$ & $10.70 \%$ & $8.19 \%$ & $8.68 \%$ & $8.18 \%$ & $9.00 \%$ \\
\hline No' deaths GM & 873 & 810 & 868 & 952 & 782 & 841 & 809 & 927 \\
\hline
\end{tabular}

Table 1: Summarizing table-period "A" vs. period "B".

The average LOS in the GM department decreased gradually from period "A" to period "B" (6.1 vs. 5.5 days) (Figure 1).

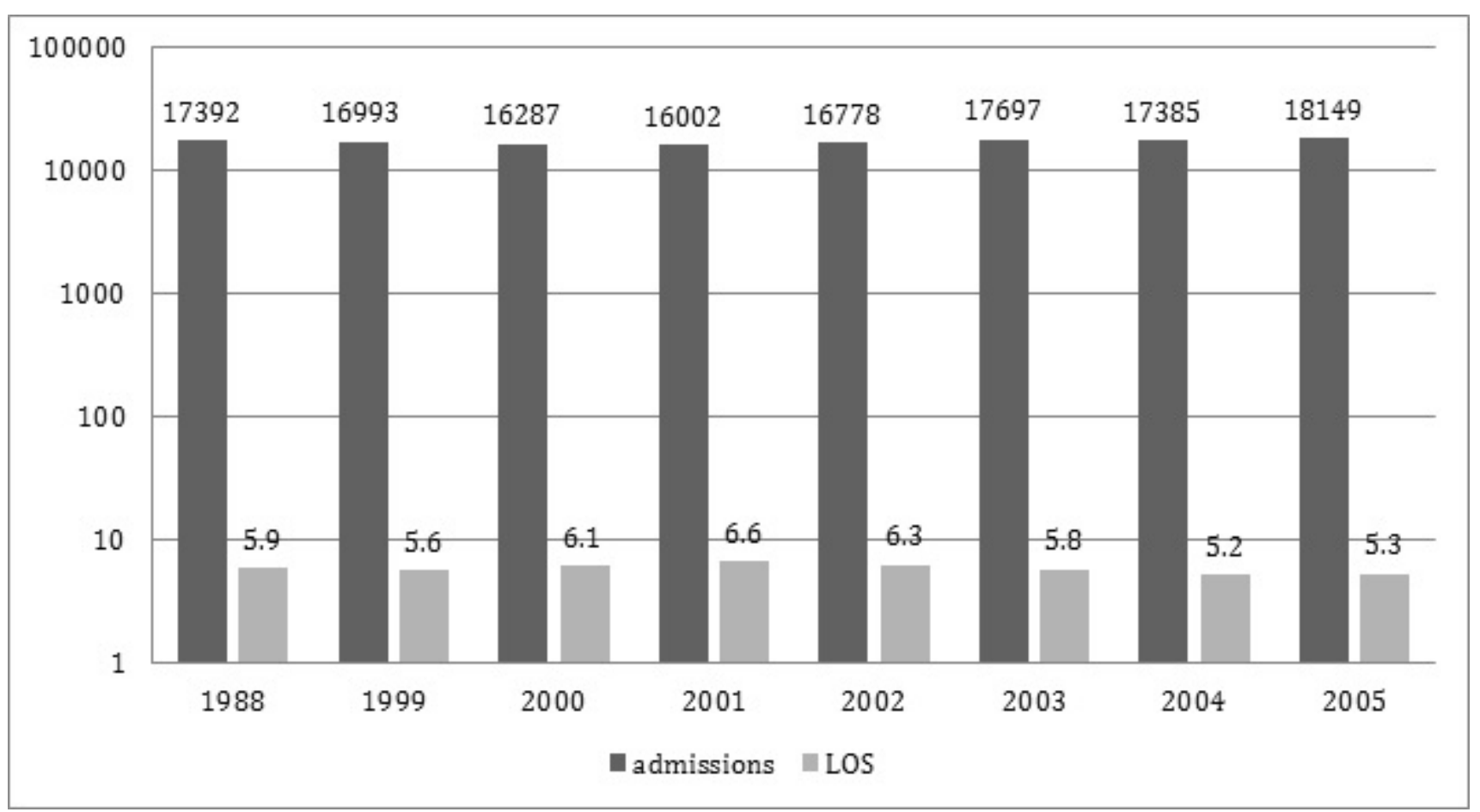

Figure 1: The LOS in the general medical wards vs. number of admissions.

The "bed rounds" i.e. the number of patients admitted per bed/year increased in parallel. Although it seems that the difference in LOS is small (0.6 days), a detailed data analysis shows a significant difference $(\mathrm{P}=0.001)$.

A detailed calculation demonstrates that a decrease in the LOS in GM departments by 0.6 days equals an increase of the GM wards capacity to admit 20 patients at every certain time.

The average LOS of patients in the ED waiting for admission to inpatient beds during period "A" was 5.05+0.98 (range 0.3 hours-99.8 hours, median 4.3 hours). It decreased significantly during period "B" to 2.45+0.21, (range 0.4-48.1 hours, median 2.5 hours) $(\mathrm{P}<0.001)$.
A geometric average of the percent of patients staying in the ED more than 10 hours from the total number of patients in the ED during period "A" was $11.5+2.4 \%$ compared to $2.6+0.7 \%$ during period "B" (Table 1). The average evaluation time was 4.2 hours +1.8 hours during period "A" compared to 3.6+1.2 hours during period "B" $(\mathrm{P}<0.001)$.

Decreasing the LOS in the GM wards during period "B" did not lead to higher rates of readmission within one week $(\mathrm{P}=0.17)$.

We compared the death rates among admitted patients into the ED in both periods using the Chi square test. We found that it was significantly higher during period "A" ( $<<0.001)$ (Figure 2$)$. 
Page 4 of 6

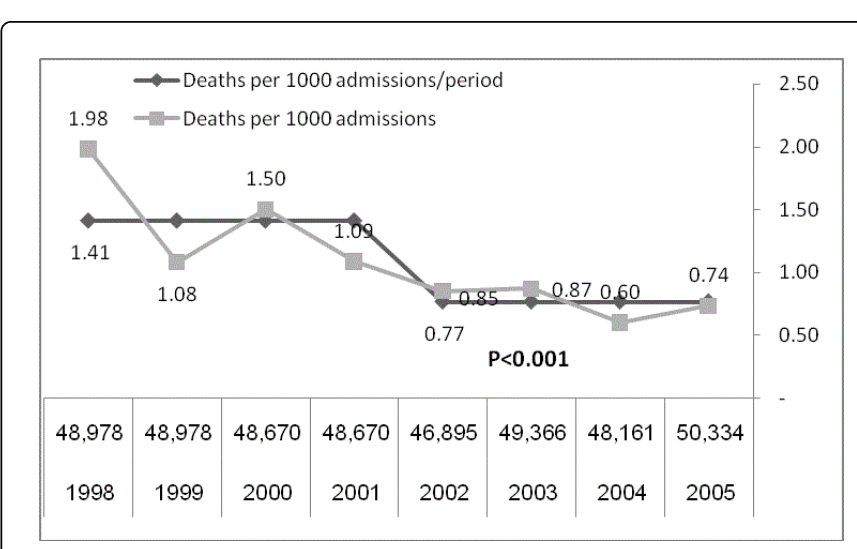

Figure 2: Death rate in the ED (1998-2001 vs. 2002-2004).

We also found a significant increase in the death rate in the GM ward during period "A" $(\mathrm{P}=0.001)$ in comparison to period "B".

\section{Discussion}

There are many causes for ED overcrowding. Although some wrote that shortage of hospital beds is the most prominent reason for ED overcrowding [6], we have shown that, without increasing the number of our hospital total number of beds, we managed to render our ED from crowded to non-crowded. New initiatives (e.g. expansion of day hospital facilities for Oncology and Hematology, Heart Failure and other patient cohorts) became prominent subsequent to the new policy, and therefore, were not a variable that distinguished Period "A" and Period "B" patterns as reported.

Furthermore, in our experience, overcrowding also is not solved by the immediate addition of extra beds, and neither by making any changes in the human resources in the ED or elsewhere. In the first trimester (Jan to Mar) of 2010, 14 beds and staff were added ad hoc to the GM wards to overcome the overburden of boarding patients in the ED. There were 2390 admissions to the GM wards in 2010 vs. 2678 in the parallel period in 2009 (a 12\% decrease). Despite the decrease in the number of admissions to the GM wards, the LOS increased from 5.5 to 6.2 day $(\mathrm{P}<0.001)$. The average daily number of patients boarding more than 8 hours in the last trimester of 2009 was $18+3$ patients while after the addition of 14 beds in the first trimester 2010, was $16+3(p=0.12)$. In a way, these facts dovetail with Parkinson's Law that says: "every task consumes the whole time it is being given to be done". In other words, the addition of beds caused a slowing of the workup in the GM departments rather than evacuating boarding patients from the ED. We also do not believe that the increase in the LOS was due to patient care becoming more complicated within one year.

There are many ways to define and calculate what an ED crowding is. Some built mathematical models to define when an ED is crowded [7-9]. Yet, in some of them there was a mismatch between the definition of overcrowding according to these calculations, and the perception of overcrowding among the ED medical and nursing staff $[7,10]$. In others, the Emergency department work index (EDWIN) has been shown to be a strong predictor of ambulance diversion, and also to correlate well with staff perception of crowding $[8,11]$.

In the Delphi study, a consensus was achieved concerning the criteria that define a crowded ED. The first is that an ED is considered crowded when less than $90 \%$ of patients have left the ED two hours post admission decision. The second criterion for ED overcrowding is when there is greater than $10 \%$ occupancy of boarders in the ED [12].

Adjacent to the definitions and criteria for "overcrowding" of ED facilities, we now add the concept of indices to measure saturation of hospital inpatient beds, and suggest that these should also be included in future formal follow up analyses of changes in hospital admission and ED policy.

It is important to clarify that the change in policy in our hospital was implemented without a priori demand estimates on the part of the hospital administration. Rather, an arbitrary threshold was set, and the observations based on implementation of policy based on this threshold constituted the basis for the observations and inferences. In addition to the explicitly clarifying this important point, we also cautiously suggest that this avoided any potential bias based on expectations arising from a priori demand estimates.

The number of acute care hospital beds in Israel was 2.0 per 1,000 residents in 2009, lower than the OECD average of 3.5 beds per 1,000 residents. The average length of stay in acute care hospitals for all causes is lowest in Israel, as in Turkey, Mexico, Norway, Denmark and the United States. The occupancy rate of curative care beds in Israel is the highest of all OECD countries, $96.3 \%$ in 2009 compared to the OECD average of $76 \%$, similar to the high rates in Canada, Norway and Ireland [13]. All these countries suffer from overcrowded EDs with patients boarding in the ED. It seems that doubling the ratio does not solve the problem (as the case in Israel vs. the UK).

According to a study performed by the Australian Institute of Health and Welfare in 1998, there was a positive correlation between the number of acute care beds and the LOS in hospitals [14]. The more beds you add to the hospital, the longer the LOS becomes. Therefore, we may conclude that the opposite way is also true: by decreasing the number of acute beds to 1.1 per 1,000 citizens, as it is in Mexico, we will further decrease the LOS in the medical wards, leaving extra workup for outpatient or community clinics [13]. This may drive the hospital work more efficiently. Logically, doubling the number of acute care bed with keeping the medical staff efficiency seems more promising in the prevention of EDs overcrowding.

In our ED, since 1995, there is a decision that patients must be transferred to inpatient beds within 4 hours. During period "B" this goal was mostly achieved while in period "A" it was not (Table 1).

During period "A", despite a gradual decline in the number of admissions to the GM wards, we found an increase in the LOS of patients in both the GM wards and the $\mathrm{ED}$, while the opposite occurred during period "B".

Many parameters influence the LOS in the GM wards and the number of boarding patients in the ED. In our study, the average number of admissions to the GM wards and the number of beds did not change significantly during both periods. Therefore, we conclude that there is a positive correlation between the LOS in the GM wards and the percent of patients boarding in the ED. The reverse might also be true; an increase in boarding time in the ED allows the departments to work less efficiently.

The mortality rate in the ED was significantly higher during period "A" in comparison to period "B". Singer et al. showed a positive correlation between the LOS in the ED and mortality rate [15]. And there was also a correlation between the boarding time in the ED and the LOS of patients in admission in the medical wards. Our study 
Page 5 of 6

supports this finding see Table 1 . We believe that the delay in treatment when boarding in the ED may complicate the patients situation, increase the LOS in the medical departments, increase the mortality rate and increase the readmissions. We also found a higher mortality rate among patients in the GM wards during period "A", despite a longer LOS. We think that the suboptimal patient treatment while boarding in the $\mathrm{ED}$, sometimes more than 3 days, positively influences the mortality rate. This point needs further investigation. It is also interesting that not only was there a decrease in the mortality rate during period " $\mathrm{B}$ " but there was also no increase in the readmission rate within one week of discharge from the wards despite the decrease in the LOS.

Jong et al. demonstrated that there are different norms between different wards within the same hospital and even within the same floor. In their study, they showed that physicians working in two hospitals with different average lengths of stay will influence the LOS of their patients according to their dominant place of work. This implies that physicians do not adapt to changing circumstances but instead bring their old work habits to their additional place of work [16]. In our hospital, in the last 5 years, 4 internal medicine departments' directors retired and they were all replaced by new directors from the existing staff. We have noticed that all of them kept the same average of patients ' LOS, as they had in the department they came from, although some had larger or smaller number of beds. This may emphasize that physicians tend to keep the same rate and efficiency of workup as they were educated before. This may explain the variability in the LOS among the four GM wards in our hospital that compose the GM department, i.e. different wards have different patterns of work and therefore different efficiency

Singer et al. showed that admitting patients to the departments' hallways increases patients' satisfaction that they were moved from the $\mathrm{ED}$, and also "creates" new beds within a short period of time, by encouraging the discharge of veteran patients [15]. Patients psychologically prefer to be admitted to the ward's hallways than staying in the ED without any specific treatment or workup. It was also shown that admitting patients to the wards' hallways does not cause any harm to those admitted patients [17].

During period "A" the ED manager considered the boarding patients in the ED as the hospital's managerial team problem. Therefore he did not force the GM to accept patients from the ED in a timely manner. Furthermore, the ED manager's attitude towards the role of the ED was that of a semi GM ward. Workup was more comprehensive and lasted many hours till making a decision.

During period "B" the managers changed the concept concerning the role of the ED. The role of the ED was redefined, into a ward where patients are evaluated specifically for their urgent conditions, using the minimum workup needed to make a safe decision for admission or discharge. And after making a decision, patients had to be moved to the GM wards within no more than 4 hrs.

Change was achieved within an eight weeks. However, daily struggles ensued for up to one year and thereafter. During the first few months, the change caused an overburdening in the GM wards. They worked even harder during the weekends. A few ward physicians tried to partially strike in the hospital. However, the hospital manager did not surrender to their pressure. Slowly, the GM wards became accustomed to the new situation. Patient workup in the GM became more efficient. Further workup was left to the community clinics. The rate of readmissions within one week did not increase.
ED overcrowding may be a whole hospital failure. However, we think the ED plays the cardinal part in that. Managers who believe that ED overcrowding is a fact will remain with overcrowded EDs. Emptying the ED needs an assertive and active manager. We agree that in our case, initially, the ED managers solved the dilemma by a winlose situation rather than a win-win situation. The ED management has to be assertive and sometimes aggressive when dealing with the GM wards. This might be considered a failure from an organizational management view of point. But a well efficient non-crowded ED attracted patients from other EDs in the city as it was during period "B".

In retrospect, we agree that the changes that we implemented must have been done gradually to allow the GM wards to ease into a new pattern of working efficiently. Extra staff might ease the initial extra work that the GM departments perceive. However, no extra beds should be added since this impedes efficiency.

We conducted a literature search to see if anyone described the hospital-wide effects of ED policy changes. Hoot and Aronsky conducted a comprehensive PubMed search and identified 93 relevant articles that dealt with ED crowding. Not one article identified the impact that ED leadership and policy has on ED crowding [5]. In our current study and in a previous one [18], we have shown that changing the policy to zero tolerance for patients boarding in the ED, accompanied by an improvement in the efficiency of patients' workup in the ED, led to ED crowd control.

\section{Limitations of the Study}

Although our finding should hold true for any hospital setting, we only have supporting data from our hospital. It would be interesting to examine the performance of EDs and the LOS in hospitals where the emergency departments are not crowded and compare them to hospitals with crowded EDs.

\section{Conclusions}

We think that an ED crowding is mainly an ED policy failure. Those who wish to manage emergency departments must be assertive and sometimes aggressive when dealing with the transfer of patients to the medical wards. In parallel, they have to assure the full backing of the hospital's administration as a condition for managing the ED. The most important factor needed for success, as in every task, is adherence to the mission, consistency, assertiveness, and a belief that the goal is attainable.

In summary: Most places accept the fact that an overcrowded ED is a norm. Until now most literature concerning ED overcrowding say that, the problem is not an ED failure but rather a whole system failure.

In this study, we would like to debunk the notion that turning a very crowded ED to a smooth running ED is mission impossible. We also show that without a change in the hospital policy, by changing the management policy in the ED a very crowded ED was rendered noncrowded within a short time, indicating that a crowded ED is not a hospital failure but rather an ED failure.

\section{References}

1. Richards JR, Navarro ML, Derlet RW (2000) Survey of directors of emergency departments in California on overcrowding. West J Med 172: 385-388. 
Citation: Basis F (2017) From an Overcrowded to a Smoothly Functioning Emergency Department within Two Months. J Gen Pract (Los Angel)

Page 6 of 6

2. Hwang U, Concato J (2004) Care in the emergency department: how crowded is overcrowded? Acad Emerg Med 11: 1097-1101.

3. Derlet RW, Richards JR (2008) Ten solutions for emergency department crowding. West J Emerg Med 9: 24-27.

4. Chan SS, Cheung NK, Graham CA, Rainer TH (2015) Strategies and solutions to alleviate access block and overcrowding in emergency departments. Hong Kong Med J 21: 345-352.

5. Hoot NR, Aronsky D (2008) Systematic review of emergency department crowding: causes, effects, and solutions. Ann Emerg Med 52: 126-136.

6. Boyle A, Beniuk K, Higginson I, Atkinson P (2012) Emergency department crowding: time for interventions and policy evaluations. Emerg Med Int 2012: 838610.

7. Reeder TJ, Garrison HG (2001) When the safety net is unsafe: real-time assessment of the overcrowded emergency department. Academic Emergency Medicine 8: 1070-1074.

8. Bernstein SL, Verghese V, Leung W, Lunney AT, Perez I (2003 ) Development and validation of a new index to measure emergency department crowding. Academic Emergency Medicine 9: 938-942.

9. Weiss SJ, Ernst AA, Nick TG (2006) Comparison of the national emergency department overcrowding scale and the emergency department work Index for quantifying emergency department crowding. Academic Emergency Medicine 13: 513-518.

10. Reeder TJ, Burleson DL, Garrison HG (2003) The overcrowded emergency department: a comparison of staff perceptions. Academic Emergency Medicine 10: 1059-1064.
11. Jones SS, Allen TL, Flottemesch TJ, Welch SJ (2006) An independent evaluation of four quantitative emergency department crowding scales. Academic Emergency Medicine 13: 1204-1211.

12. Beniuk KO, Boyle AA, Clarkson PG: Emergency department crowding: prioritising quantified crowding measures using a Delphi study. Emerg Med J 29: 868-871.

13. (2011) OECD Health at a Glance: Key findings for Israel.

14. Acute care beds and length of stay at.

15. Singer AJ, Thode HC Jr, Viccellio P, Pines JM (2011) The association between length of emergency department boarding and mortality. Acad Emerg Med 18: 1324-1329.

16. de Jong JD, Westert GP, Lagoe R, Groenewegen PP (2006) Variation in hospital length of stay: do physicians adapt their length of stay decisions to what is usual in the hospital where they work? Health Serv Res 41: 374-394.

17. Viccellio AW, Santora C, Singer AJ, Thode HC Jr, Henry MC (2009) The association between transfer of emergency department boarders to inpatient hallways and mortality: a 4-year experience. Ann Emerg Med 54: 487-491.

18. Basis F, Pollack S, Utits L, Michaelson M (2005) Improving the pattern of work towards emergency medicine at the Emergency Department in Rambam Medical Centre. Eur J Emerg Med 12: 57-62. 